\title{
Manipulation under anaesthesia versus lysis of adhesions for arthrofibrosis of the knee: a 6-month randomized, multicentre, non-inferiority comparative effectiveness protocol
}

\author{
Ling Zhang ${ }^{1}$, Mengning Yan ${ }^{2}$, Shiyi Chen ${ }^{3}$, Yinghui Hua ${ }^{3}$, Zimin Wang ${ }^{4}$, Lili Xu ${ }^{1}$, Shuai Fan ${ }^{1}$ Lu Lin ${ }^{5}$, \\ Bin $\mathrm{Cai}^{1}$
}

${ }^{1}$ Department of Rehabilitation, Shanghai Ninth People's Hospital, Shanghai Jiao Tong University School of Medicine, Shanghai, China; ${ }^{2}$ Department of Orthopaedic Surgery, Shanghai Ninth People's Hospital, Shanghai Jiao Tong University School of Medicine, Shanghai, China; ${ }^{3}$ Department of Sport Medicine, Huashan Hospital, Fudan University, Shanghai, China; ${ }^{4}$ Department of Orthopaedic Surgery, Changhai Hospital, The Second Military Medical University, Shanghai, China; ${ }^{5}$ Clinical Research Center, Shanghai Ninth People's Hospital, Shanghai Jiao Tong University School of Medicine, Shanghai, China

Contributions: (I) Conception and design: S Chen, B Cai; (II) Administrative support: L Zhang, L Xu; (III) Provision of study materials or patients: Y Hua, Z Wang, M Yan; (IV) Collection and assembly of data: L Zhang, L Xu, Y Hua, Z Wang, M Yan; (V) Data analysis and interpretation: S Fan, L Lin; (VI) Manuscript writing: All authors; (VII) Final approval of manuscript: All authors.

Correspondence to: Bin Cai. Department of Rehabilitation, Shanghai Ninth People's Hospital, Shanghai Jiao Tong University School of Medicine; 639 Zhizaoju Road, Huangpu District, Shanghai 200011, China. Email: shrehab@163.com.

Background Recent studies show similar outcomes between lysis of adhesions (LOA) and manipulation under anaesthesia (MUA) in patients with arthrofibrosis within 3 to 6 months after arthroscopic knee procedures. As MUA offers positive efficacy with less expense and more convenience, the first consideration in clinical practice shifting to MUA may save much medical cost when MUA is non-inferior to surgery. There is a lack of evidence comparing the clinical outcomes of these two surgical techniques. The purpose of our study is to determine whether MUA is non-inferior to LOA for improving clinical outcomes in patients with loss of flexion within 3 and 6 months after arthroscopic knee surgery.

Methods: This multicentre randomized controlled trial (RCT) will recruit 360 patients within 3 to 6 months after arthroscopic knee surgery with a magnetic resonance imaging (MRI)-confirmed arthrofibrosis to prove non-inferiority of MUA. Patients are randomly assigned to the LOA group ( $\mathrm{n}=180)$ or the MUA group $(\mathrm{n}=180)$ in a $1: 1$ ratio with random sequences using the SPSS Statistics V.22. The standard surgical procedures will be performed within 2 weeks after randomization, and each patient will receive unified perioperative physical therapy $(\mathrm{PT})$. The primary outcome measure is the range of knee flexion. Secondary outcome measures include physical function, activity level, general health, knee pain, and the occurrence of adverse events. Safety measures include physiological parameters (i.e., blood pressure, pulse, respiratory rate) and complications (i.e., component damage, patellar tendon tear and fracture during surgical procedures, neurological or internal medicine conditions, venous thromboembolism, bleeding and infection postoperatively). The noninferiority margin is defined as a difference of 10 degrees on the range of knee flexion between groups and was assessed with a one-sided $\alpha$ of .025 . To investigate relative effectiveness between the groups, linear mixed-effects model will be used for continuous data, and generalised estimating equation for dichotomous data. Analyses will follow the intention-to-treat principle.

Discussion: Findings of our study will help clinicians determine the risk-benefit balance of MUA and LOA, two frequently used surgical procedures for patients with knee arthrofibrosis.

Trial registration: Chinese Clinical Trial Registry (www.chictr.org.cn); ChiCTR2000033467.

Keywords: Knee; arthrofibrosis; loss of motion; adhesiolysis; arthroscopy; manipulation under anaesthesia (MUA) 
Submitted Jan 08, 2021. Accepted for publication Mar 23, 2021.

doi: 10.21037/apm-21-48

View this article at: http://dx.doi.org/10.21037/apm-21-48

\section{Introduction}

Arthrofibrosis of the knee is a common complication after arthroscopic knee surgery, with varied incidence according to the type of initial surgery (1). Recent studies reported a $2-5 \%$ incidence of arthrofibrosis in patients who underwent isolated anterior cruciate ligament (ACL) reconstruction (2-4). Noyes et al. (5) reported that as many as $23 \%$ of patients who underwent knee multiple ligament reconstruction developed arthrofibrosis. Traumatic knee dislocation resulted in the highest incidence of postoperative arthrofibrosis, as high as $57 \%$ (6). The estimated incidence of arthrofibrosis following arthroscopic knee surgery varied from $4 \%$ to $35 \%$ (7). Loss of motion due to arthrofibrosis decreases the patients' ability to perform activities of daily living and contributes to poor knee joint function, which is a frustrating complication for both the surgeon and patient.

Given the high proportion of patients who do well with non-surgical options, a trial of at least 1 month of conservative management has been recommended before considering manipulation under anaesthesia (MUA) or lysis of adhesions (LOA) for refractory cases (8-10). LOA has been a long-standing treatment for knee arthrofibrosis (11). Arthroscopy is minimally invasive and allows the visualization of intraarticular structures, thereby providing good results with low incision-related complication rates $(1,12)$. Despite these positive effects, LOA is notorious for the various complications, including hemarthrosis, infection, pulmonary embolism, and heterotopic bone formation. The LOA procedure is difficult to perform due to joint space narrowing and has high requirements for surgical techniques and equipment $(9,13)$. Recent studies of the success following LOA for knee arthrofibrosis have demonstrated acceptable results across objective and subjective outcomes $(9,12)$.

Besides LOA, a variety of other less invasive alternatives have been suggested in an attempt to treat refractory knee arthrofibrosis (14). Similar to LOA, MUA has been widely reported to achieve improvement in knee flexion and patient-reported outcomes in patients with knee arthrofibrosis resistant to conservative treatment (15). Nevertheless, there is wide inconsistency among authors regarding the timing of MUA. The most common time point was approximately 90 days after the prior knee surgery
(14,16-18), and recent studies recommended MUA within 3 months of arthroscopic knee procedures (18). However, several studies that assessed the efficacy of MUA performed between 3 and 6 months after knee surgery indicated that the efficacy of MUA was not affected by such a delay $(16,17)$.

A systematic review indicated that MUA is more effective during the first six months because there is less adhesive tissue in the knee and less mature tissue is easier to overcome with manipulation $(19,20)$. Clinical practice guidelines vary regarding the effectiveness and time of MUA for the arthrofibrotic knee (19,21), with a recent clinical practice guideline providing that MUA is the firstline operative treatment of choice for patients that fail physiotherapy and continue to experience functionally limiting knee flexion (22). MUA is frequently used as either an alternative, or adjunct, to an LOA for patients with loss of flexion within 6 months after arthroscopic knee procedures (22). The MUA procedure is easily performed and costs far less than LOA (23). The economic analysis showed that the mean cost of LOA was $£ 2,170.46$, and the average duration of LOA was 76.61 minutes, however, the mean cost of MUA was $£ 424.81$, and the average duration of MUA was 25.11 minutes (24). MUA is suggested to be the most cost-effective option compared with physiotherapy and LOA (24). Given that LOA is likely associated with a higher complication rate, technical-demand, and cost, it would be necessary to determine if there is any difference in outcomes between these 2 procedures. Until recently, few studies have directly compared the clinical outcomes of these 2 surgical procedures. The main purpose of this study is to determine whether MUA is non-inferior to LOA for improving clinical outcomes in patients with loss of flexion within 3 and 6 months after arthroscopic knee surgery.

\section{Specific aims}

\section{Aim 1}

To compare the 6-month improvement of knee flexion between patients that underwent LOA and those that underwent MUA.

\section{Aim 2}

To compare self-reported outcomes of knee pain and 
function between groups during the 6-month follow-up period.

\section{Aim 3}

To evaluate the cost-effectiveness of LOA and MUA for patients with loss of flexion during the 6-month follow-up period.

We present the following article in accordance with the SPIRIT reporting checklist (available at http://dx.doi. org/10.21037/apm-21-48).

\section{Methods}

\section{Study design}

This study is a multicentre, noninferiority randomized controlled trial performed in China. The trial protocol was designed using the Standard Protocol Items: Recommendations for Interventional Trials (SPIRIT) Statement (25). The results will be reported according to the Consolidated Standards of Reporting Trials (CONSORT) Statement (26). This RCT will be carried out according to the Declaration of Helsinki (as revised in 2013) and has been approved by the Medical Research Ethics Committee (SH9H-2020-T218-1). Informed consent will be obtained from each patient before taking part. This study has been registered at chictr.org.cn (ChiCTR2000033467) before the start of inclusion.

\section{Trial procedures}

The first patient was included on 30 August 2020. Patients are being recruited from the orthopaedic clinic of three hospitals in China (Shanghai Ninth People's Hospital, Huashan Hospital, Changhai Hospital). According to specific requirements and qualifications, these hospitals are selected, and the study protocol has been provided before including first patient. Eligible patients are randomly assigned to receive either LOA or MUA at these hospitals. To be included in the study, the surgeon has to understand the study design, review patient inclusion criteria, and complete two training sessions that introduce standardized surgical procedures that have been reported previously $(9,27)$. Postoperative physical therapy (PT) is performed at the PT clinic of three participating hospitals. These PT clinics will be contacted by the researcher to provide them the PT program before the start of the trial.

\section{Participants}

Patients between 18 and 50 years with knee arthrofibrosis within 12 to 24 weeks following unilateral arthroscopic knee surgery are being recruited from the orthopaedic clinic of these hospitals. Eligible patients meet an international consensus on the definition of fibrosis of the knee joint (28) and have an magnetic resonance imaging (MRI)-confirmed knee arthrofibrosis, with the range of active knee flexion $<100^{\circ}$. Exclusion criteria are as following:

* Knee extension deficit $>5^{\circ}$;

- Postoperative infection (septic arthritis) or heterotopic ossification;

* Loss of motion caused by ligament reconstruction, or metal hardware;

* Previous knee arthroplasty on the index knee;

* Other causes affecting the function of lower limbs;

* Unable to complete the questionnaires.

\section{Patient recruitment}

All patients with knee arthrofibrosis who visit the orthopaedic clinic will be screened for eligibility. A potential patient is informed verbally about the study by the surgeon during the first outpatient visit. Training will be delivered to directors in orthopaedic outpatient clinics at the participating sites to inform them of the study and regarding screening patients for eligibility. Providers in orthopaedic clinics will have copies of the screening form to screen potential patients according to the inclusion and exclusion criteria. Informed consent is signed prior to randomization.

\section{Randomization and blinding}

After signing informed consent, patients are randomly assigned to the LOA group or MUA group. Random numbers generated by the SPSS Statistics V.22 are arranged in ascending order with 1-180 defined as the treatment (LOA) group and 181-360 as the control (MUA) group. Stratification occurs using a predetermined cut-off for the range of flexion; those who present with equal to, or greater than $90^{\circ}$ are defined as "mild", those with between $70^{\circ}$ and $89^{\circ}$ as "moderate", and those with less than $70^{\circ}$ as "severe" (28). Randomization are stratified by participating sites, the range of flexion, and age (18-40 and 41-50 years old). An independent research assistant who is not involved in enrolling and assigning participants, evaluation and 
intervention generates a randomisation schedule for all participants at participating sites. A separate researcher will communicate with the study participants, surgeons, physiotherapists and sites.

Participants and physicians are not blinded. A researcher will collect all baseline data; the outcome assessor and physiotherapists will be blind to the participant's stratification and study hypothesis. Statisticians will be blinded to the group of assignments during analysis. After completing the analysis, data will be unblinded for the interpretation of the results. When performing the informed consent process, participants will be informed that both interventions are evidence-based care, but will be kept blind to their stratification and study hypothesis. Outcome expectations of participants for each treatment will be assessed prior to randomization.

\section{Interventions}

\section{LOA procedures}

LOA is performed within 2 weeks after randomization by surgeons good at arthroscopic surgery. The principle of the LOA is to remove the adhesions in the suprapatellar pouch, infrapatellar fat pad, the intercondylar notch, and around the patella to the greatest extent possible so as to facilitate normal knee motion. The patient with lateral thigh support is in the supine position. Surgery will be performed under general anaesthesia. With the patient's consent, photographs of knee flexion with the patient under anaesthesia will be taken to record the preoperative range of knee flexion. The anterolateral (AL) and anteromedial (AM) portals are approximately $5-\mathrm{mm}$ incisions that are established in accordance with standard knee arthroscopy portal sites. AL and AM portals are used for inspection of the knee joint and debridement of intraarticular adhesions. In cases of knee flexion deficits, it is necessary to release the space between patellar tendon and anterior tibial plateau. If the knee cannot be flexed past $70^{\circ}$, the arthroscopy starts with the superolateral (SL) portal and the superomedial (SM) portal. Anterior adhesions within peripatellar region and the suprapatellar pouch will be cleared or ablated using radiofrequency $(\mathrm{RF})$ energy through the SL and SM portals. The remaining adhesions in the infrapatellar fat pad and the intercondylar notch will be removed using an arthroscopic shaver and $90^{\circ}$ cautery through the AM portal. This allows for the improvement of patella infera and patellar mobilization. All intraarticular adhesions of the medial and lateral gutters are removed through a motorized shaver.
Capsular or lateral retinacular release will be performed if necessary. Taking intraoperative images of knee flexion will be required to document post-release range of motion (ROM) (with the patient's consent). This helps to compare with preoperative $\mathrm{ROM}$ and to make a target-oriented rehabilitation program after the surgery.

\section{MUA procedures}

MUA is performed within 2 weeks after randomization by the orthopaedic surgeons good at knee surgery. The patient with lateral thigh support is in the standard supine position. Surgery will be performed under general anaesthesia. With the patient's consent, photographs of knee flexion with the patient under anaesthesia will be taken to measure preoperative ROM. The surgeon performs mobilization of the patella to start the manipulation of the knee, which aids with mediolateral shift and inferior displacement. The proximal tibia will be used as a lever in combination with mobilization of the tibiofemoral joint to break up the adhesive tissues and achieve maximal flexion progressively and gradually. Slight pressure will be applied on the tibial tubercle to avoid disruption of the extensor mechanism. Gentle manipulations of the knee will be performed to obtain $\geq 110^{\circ}$ of knee flexion until resistance is felt by the surgeon to prevent further complications. Taking intraoperative pictures will be required to record postrelease knee flexion ROM with the patient's consent.

\section{Physical rehabilitation procedures}

All patients receive an information letter with preoperative instructions and formal education on the benefits of perioperative rehabilitation. The flow of participants through the trial is illustrated in Figure 1. Patients are referred to the PT clinics for postoperative rehabilitation. The treatment protocol consists of 2 weeks of inpatient rehabilitation, 6 weeks of outpatient rehabilitation, and 8 weeks of home exercise given by a knee-specialized physiotherapist. As pain killers or any inflammatory drug are not given post-operatively which often is prescribed routinely after surgical procedures, pain management is essential to allow for maximum participation in the rehabilitation program, including patient-controlled analgesia (PCA), continuous cryotherapy and compression (CCC) and continuous passive motion (CPM) within 48 hours after surgery. The PT programme follows a standardized rehabilitation protocol and initiates on the third day after surgical procedures. Despite the abundant literature on PT for arthrofibrosis of the knee after surgical 


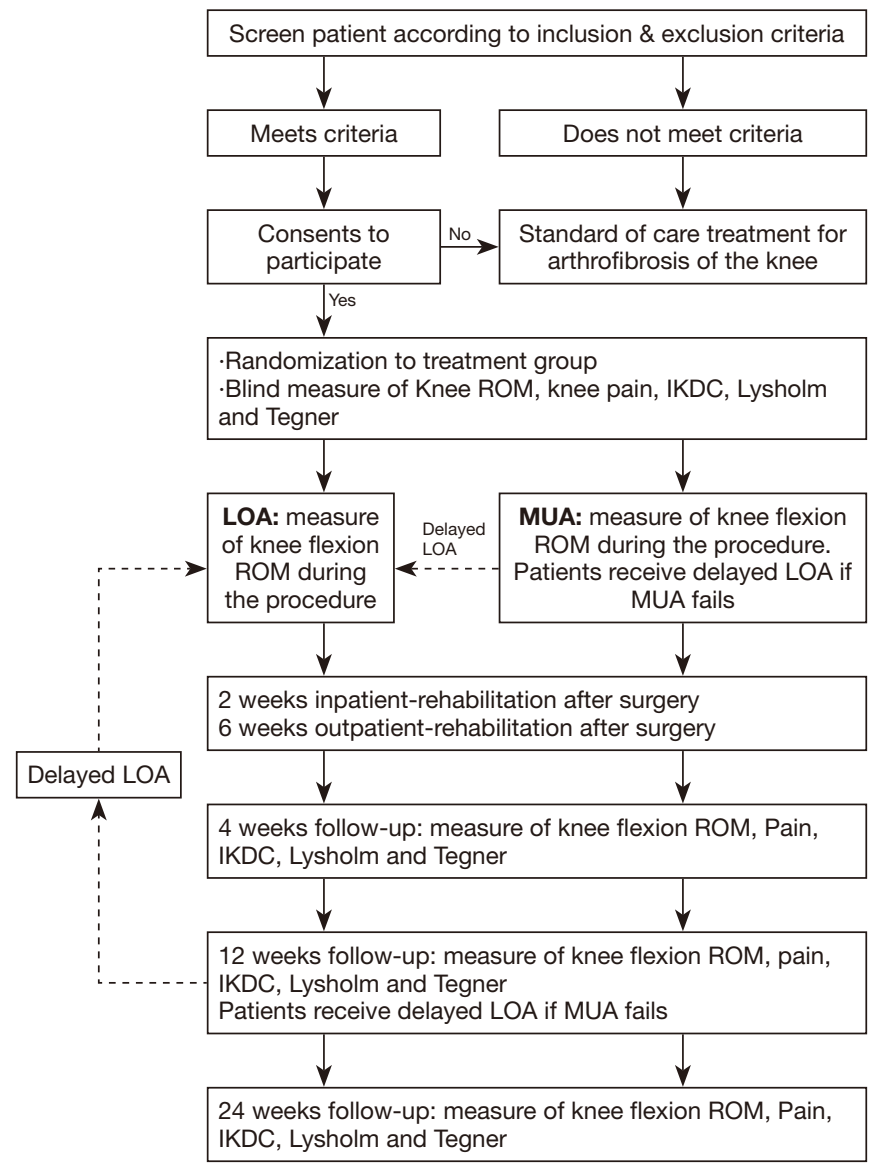

Figure 1 Participants recruitment and flow of the study. MUA, manipulation under anaesthesia; LOA, lysis of adhesions; ROM, range of motion; IKDC, International Knee Documentation Committee.

release, there is currently no gold standard. Notably, quadriceps muscle activation and knee ROM exercises are initiated immediately after surgery and aggressively advanced within the limits of patient tolerance (27). Once sufficient muscle activation and knee motion are obtained, the patient will resume the criterionbased postoperative rehabilitation protocol (see online Supplementary appendix 1) at the caution of the treating physical therapist (27). The protocol includes phased knee ROM, lower limb strengthening, neuromuscular control, and an agility program. Participants are assessed before and after treatment sessions to determine the effects of treatment modalities. If participants do not respond to ongoing manual techniques and exercises, we will adjust the rehabilitation program. All patients will be monitored postoperatively until they have completed a 6-month follow-up.

Stop rule conditions are as following: patients requiring to withdraw without reasons; intraoperative knee flexion under general anaesthesia $<110^{\circ}$ during LOA procedures; active knee flexion at 3 -month follow-up after LOA $<110^{\circ}$; patients suffering from other knee injuries or procedures that affect the results; occurrence of serious adverse events (SAEs).

\section{Cross-over}

Based on physical examination and the range of knee flexion, the surgeons will decide whether MUA has been 
successful. When MUA has failed (e.g., intraoperative knee flexion under general anaesthesia $<110^{\circ}$ or active knee flexion at 3 -month follow-up $<110^{\circ}$ ), a delayed LOA can be performed. If the worsening of symptoms is demonstrated or participants request to stop treatment, providers at these sites will re-evaluate the situation and may adapt the treatment to meet the patients' needs. Patients are not forbidden from using medications; however, the use of these medications will be documented and reported.

\section{Outcome measures}

\section{Primary outcome}

The primary outcome is the change in the range of knee flexion from baseline to 6 months measured by a plastic goniometer with $25-\mathrm{cm}$ movable arms in $1^{\circ}$ increment. It has been shown to be reliable if the participant remains in the same position for the measurement (29). With patients lying supine on an examination table, measurements of knee flexion will be taken in active and maximum flexion of the knee with the hip flexed. One arm of the goniometer will be placed along the greater trochanter and the lateral epicondyle of the femur and the other arm will be aligned with the fibular head and the lateral malleolus of the fibula.

\section{Secondary outcomes}

The secondary outcomes to evaluate clinical effectiveness are:

(I) Change in:

(i) Self-reported knee function, measured by the International Knee Documentation Committee (IKDC) with good validity and reliability for the evaluation in the Chinesespeaking population (30-32);

(ii) Self-reported instability and function, measured by Lysholm knee score with good validity and test-retest reliability for Chinesespeaking population $(33,34)$;

(iii) Level of activity, measured by Tegner Activity Scale (TAS) with good validity and reliability for the Chinese population (35);

(iv) Quality of life, measured by EuroQol 5 Dimensions 5 Level Survey (EQ-5D-5L) with good validity and reliability for the Chinese population $(36,37)$;

(v) Pain, measured with the visual analogue scale (VAS) at rest and during weight- bearing;

(vi) Percentage of cross-over; the number of patients receiving delayed LOA.

(II) Patient's preference on the two treatments; patient rating of satisfaction of the intervention; relation between a patient's expectation and their satisfaction.

(III) Surgeon's impression of the location and maturity of scarring in the arthrofibrotic knee, the physical examination findings, and the meniscal and articular cartilage findings during LOA procedures and their surgical management of these findings.

(IV) The 6-month cost of knee-related health care utilization.

(V) Adverse events.

Safety measures include physiological parameters (i.e., blood pressure, pulse, respiratory rate) and complications (i.e., component damage, patellar tendon tear and fracture during surgical procedures, neurological or internal medicine conditions, venous thromboembolism, bleeding and infection postoperatively). Follow-up examinations will be performed at the orthopaedic outpatient clinics of participating hospitals at 1, 3, and 6 months after surgery. The influence of possible prognostic factors including age, sex, body mass index (BMI), using tobacco, type of prior surgery, immobilization time, preoperative knee ROM, and time from arthroscopic knee surgery to MUA or LOA on the clinical outcomes will be analysed during the follow-up period. These putative factors have been selected based on current knowledge of prognostic factors of LOA for knee arthrofibrosis that have been reported in previous studies (1).

\section{Adherence and safety evaluation}

At first, each patient will be informed of detailed study content and benefits; they have the freedom to decide whether to participate. To promote patient retention, the study coordinator at these centres will inform patients that although they have the right to withdraw at any time, dropping out without reason reduces the ability to answer the research question and therefore weakens the study. For those patients who meet inclusion criteria but refuse to participate in the study, the standard of care treatment will be given. If patients have trouble complying with the intervention or completing follow-up testing, they will be encouraged to discuss these challenges with the study coordinator. If patients miss measurement appointments, up to three reminders will be sent and, if necessary, the patient will be contacted by telephone to reschedule a convenient time. The received surgical procedures and the number 
of postoperative PT will be recorded and reported, as well as any problems or adverse events related to the trial, including time, severity, duration, solutions and outcomes.

\section{Sample size calculation}

The sample size was calculated based on a standard deviation of 19 degrees on knee flexion ROM [retrieved from the study of Sassoon at al. (17)], a power of $90 \%$ and an $\alpha$ level of 0.05 . To determine the noninferiority margin, we systematically reviewed articles that reported the outcomes of LOA for patients with arthrofibrosis after arthroscopic knee surgery. Meta-analysis was performed to construct confidence intervals. Improvement of knee flexion ROM was $45.2^{\circ}$ for patients with arthrofibrosis who underwent LOA (95\% CI: 42.1-48.3). A noninferiority threshold of 10 degrees on knee flexion ROM was determined by an expert panel corresponding to an effect size $f$ of 0.2 . With an anticipated $10 \%$ loss at the 6-month follow-up and 5\% delayed LOA, 180 participants per group, or a total of 360 participants are needed in this non-inferiority trial. Sample size calculation was performed using G*Power software, V.3.1. Recruitment did not go ahead without prior institutional review board approval.

\section{Data analysis}

\section{Effectiveness analysis}

Shapiro-Wilk and Kolmogorov-Smirnov statistics will be used to test the normality assumption of distributions of measured variables, and Levene's test will be used to examine the homogeneity of variance assumption. Chisquare analyses (categorical data) and the independent Student's $t$-test (continuous data) will be used to examine baseline differences between groups. To investigate relative effectiveness between the groups, linear mixed-effects models will be used for continuous data, and generalised estimating equation for dichotomous data. All analyses will follow the intention-to-treat principle.

In primary linear mixed model, the model with a fourlevel structure (treatment, participant, hospital and time) will be analysed to investigate the overall between-group differences. In secondary linear mixed models, the outcome variables (e.g., physical function, level of activity) will be analysed in a similar way. The baseline variables are treated as a covariate in all models.

Additionally, potential confounders will be added as covariates (e.g., age, sex, BMI, using tobacco, type of prior surgery, immobilization time after the initial procedure, preoperative knee ROM, and the time from arthroscopic knee surgery to MUA or LOA). Covariates are considered as contributing to more than $10 \%$ change in those estimated parameters of time-by-treatment interaction, as previously reported (38).

Analyses will follow the as-treated principle, in which participants will be analysed according to their adherence to treatment allocation, including three groups: (I) patients assigned to receive LOA who receive LOA, (II) patients assigned to receive MUA who complete MUA without having LOA at follow-up, and (III) patients assigned to receive MUA who have delayed LOA during follow-up. Patients who are assigned to receive LOA or MUA but do not have surgery will be excluded from the as-treated analysis.

A $97.5 \%$ CI will be used for the outcome of knee flexion ROM, and a 95\% CI for the secondary outcomes. Noninferiority will be showed when the noninferiority margin is not included in the $97.5 \%$ CI. Sensitivity analysis will be conducted to assess the robustness of the results with different assumptions regarding missing values. We prespecify the use of our statistical model as the primary plan for handling missing data, and we will perform many more imputations using the Markov chain Monte Carlo method in sensitivity analyses (39). A value of $\mathrm{P}<0.05$ is considered to be significant. All statistical analyses will be performed using SPSS Statistics software (v 24; IBM, Corp. Software, Armonk, NY, USA). A statistician will be responsible for all longitudinal analysis.

\section{Cost-effectiveness analysis}

Effect measures in the economic evaluation are the range of knee flexion and general health based on the EuroQol. The analysis will be performed according to the intentionto-treat principle. A full cost-effectiveness and cost-utility analysis will be carried out. Incremental cost-effectiveness ratios (ICERs) are calculated according to the difference in mean effects and mean total costs between groups.

\section{Data handling and confidentiality}

Data from each participant will be recorded in forms in the participant data folder, which will be labelled with study numbers according to the number from the randomization lists. The key to all participant numbers is only accessible for researchers and the principal investigators. Outcome data is anonymised and only available to the associated 
researchers. Folders will be filed sequentially by number and maintained in a locked filing cabinet in a locked room. A research assistant will transfer all data into the master data spreadsheet. Accuracy of data is guaranteed by secondary review by co-authors. Data will be collected and stored for 10 years. Intervention groups will be numbered 1 or 2 because statistical analyzers who perform the statistical analysis will be blinded to the group of assignments. Original data will be saved in a database at the initiating hospital (Shanghai Ninth People's Hospital), and a spreadsheet will be stored on a portable drive.

To ensure security, data input capabilities are only accessible for the researcher assistant (L Zhang) and the coordinating investigator $(\mathrm{L} \mathrm{Xu})$. Data processing capabilities are only accessible for the principal investigators, statisticians (L Lu and S Fan), and authorized research staff.

\section{Trial organisation and monitoring}

No official steering committee is included in this study. The research team will consist of the authors listed in this article, in addition to physiotherapists and administrative staff at each site who will assist with the entire process of the study and data entry. All adverse events or problems related to this study will be recorded and reported to the ethical committee. The principal investigators ( $Z$ Wang, Y Hua and M Yan) will manage the study flow and perform inspections of the enrolment, treatment, and procedures throughout the entire study. Other investigators will monitor data collection and facilitate data integrity performed with periodic evaluations continually during the data collection phase. The primary researchers (B Cai and S Chen) will participate in the discussion of all data analyses before the final presentation of these results. The data supporting the results are available on request from the corresponding author CB.

\section{Patient and public involvement}

No patients were involved with the design, data provision, analysis or publication of the study.

\section{Etbics and dissemination}

This RCT will be performed in accordance with the Declaration of Helsinki (as revised in 2013) and has been approved by the Ethics Committee (reference number:
SH9H-2020-T218-1). Informed consent will be obtained from each patient before taking part. The results will be reported following the CONSORT statement at scientific conferences or in a peer-reviewed source. An ethics review protects human medical research participants to ensure compliance with federal regulations. Any modifications to the protocol which may impact study procedures or the conduct of the study will require approval by the Institutional Review Board and a formal amendment to the protocol. This clinical trial has been registered at www.chictr.org.cn with a registration number ChiCTR2000033467. Patients included in this study have right to withdraw at any time, and the reasons should be documented. The research coordinator will report all SAEs within 24 hours of observation through the online submission system to the ethics committee.

\section{Discussion}

In this noninferiority, randomized study, the effects of two surgical techniques are compared concerning clinical outcomes and the cost-effectiveness in patients with loss of flexion within 3 and 6 months after arthroscopic knee surgery. It is clinically significant because of a lack of evidence regarding the treatment of arthrofibrosis after arthroscopic knee surgery.

The effectiveness of LOA for patients with arthrofibrosis of the knee within 6 months after initial procedures have been shown in the previous studies $(17,18)$. Diagnostic arthroscopy performed during LOA allows visualization of intra-articular scarring $(9,12,13)$. Arthroscopy also allows the release of adhesions in the suprapatellar pouch, medial and lateral gutters, infrapatellar fat pad, and intercondylar notch, providing greater patellar mobility and patella infera (9). However, it may result in complications such as wound dehiscence, infection, and heterotopic bone formation. By contrast, MUA procedures achieve similar clinical outcomes with decreasing surgical risks and requiring the patient to undergo shorter in-hospital treatment. MUA procedures are often easier, less expensive, less technically-demanding and more convenient for patients. Nevertheless, there is wide inconsistency among authors regarding the timing of MUA, and the published evidence does not support the notion that MUA may be considered an alternative to LOA for patients with arthrofibrosis within 3 to 6 months after arthroscopic knee surgery. The results of this study may assist the creation of best practice guidelines for patients with arthrofibrosis of the knee. 
It is worth noting that early gains in ROM soon after surgical procedures are not an indicator of the final results. Ipach et al. (40) reported that substantial decreases in ROM 6 weeks after MUA or LOA may be found due to an abnormal inflammatory response or a lack of PT. Therefore, postoperative rehabilitation is crucial for the successful clinical outcomes of surgical procedures for knee arthrofibrosis. A ROM gain intraoperatively may be lost because of a prolonged period of immobility due to postoperative pain. In particular, the results of the two treatments highly depend on pain control postoperatively, patient motivation and compliance with postoperative rehabilitation protocols. Appropriate management of pain after surgery is vital to decrease inflammation, to accelerate the progress of physical rehabilitation, and to prevent recurrent stiffness $(9,41)$.

However, the recruitment method may have biased the trial sample toward patients more likely to benefit from MUA and may have influenced patients' perception of the interventions. This study may have a bias for blinding of participants and surgeons, and a bias for incomplete outcome reporting. Various measures will be taken to minimise bias and ensure trial validity. Patients will be informed that both interventions are evidence-based care, and those who want only MUA will be excluded at the time of recruitment. All patients will be provided with standardised surgical procedures, physiotherapy, and written home exercises advice they need to perform. Since not all patients may be evaluated at each time point (missing data), sensitivity analysis will be performed to assess the robustness of the results.

The results of this study will help clinicians determine the risk-benefit balance of these two frequently used surgical procedures. Therefore, the clinical relevance of this study is very high since MUA may be an alternative to LOA for patients with loss of flexion within 3 and 6 months after arthroscopic knee procedures. Finally, further work is needed to directly compare the effectiveness and costeffectiveness of these two surgical techniques in a constant patient population, including not only the cost of the procedures, but therapy costs, the amount of lost time from gainful work, and other costs over the complete recovery period.

\section{Conclusions}

This trial will generate detailed and accurate evidence on the efficacy and safety of MUA and LOA for the treatment of patients with loss of flexion within 3 and 6 months after arthroscopic knee surgery. Furthermore, confirmation of the hypothesis may help clinicians determine the risk-benefit balance of these two frequently used surgical procedures in the management of patients with knee arthrofibrosis.

\section{Trial status}

Recruitment was started on 30 August 2020 and is estimated to be completed in August 2022.

\section{Acknowledgments}

This manuscript has been edited for English language by professional editors at Editage, a division of Cactus Communications.

Funding: This study is supported by the (China) State's Key Project of Research and Development Plan (grant numbers 2018YFF 0300504).

\section{Footnote}

Reporting Checklist: The authors have completed the SPIRIT reporting checklist. Available at http://dx.doi.org/10.21037/ apm-21-48

Conflicts of Interest: All authors have completed the ICMJE uniform disclosure form (available at http://dx.doi. org/10.21037/apm-21-48). The authors have no conflicts of interest to declare.

Ethical Statement: The authors are accountable for all aspects of the work in ensuring that questions related to the accuracy or integrity of any part of the work are appropriately investigated and resolved. This RCT will be performed in accordance with the guidelines outlined in the Declaration of Helsinki (as revised in 2013) and has been approved by the Ethics Committee of Ninth People's Hospital, Shanghai Jiao Tong University School of Medicine, Shanghai, China (reference number: SH9H2020-T218-1). Informed consent will be obtained from each patient before taking part. The results will be reported following the CONSORT statement at scientific conferences or in a peer-reviewed source.

Open Access Statement: This is an Open Access article distributed in accordance with the Creative Commons Attribution-NonCommercial-NoDerivs 4.0 International 
License (CC BY-NC-ND 4.0), which permits the noncommercial replication and distribution of the article with the strict proviso that no changes or edits are made and the original work is properly cited (including links to both the formal publication through the relevant DOI and the license). See: https://creativecommons.org/licenses/by-nc-nd/4.0/.

\section{References}

1. Kim YM, Yong BJ. Prognostic factors of arthroscopic adhesiolysis for arthrofibrosis of the knee. Knee Surg Relat Res 2013;25:202-6.

2. Harner CD, Irrgang JJ, Paul J, et al. Loss of motion after anterior cruciate ligament reconstruction. Am J Sports Med 1992;20:499-506.

3. Magit D, Wolff A, Sutton K, et al. Arthrofibrosis of the knee. J Am Acad Orthop Surg 2007;15:682-94.

4. Mayr HO, Brandt CM, Weig T, et al. Long-term Results of Arthroscopic Arthrolysis for Arthrofibrosis After Anterior Cruciate Ligament Reconstruction. Arthroscopy 2017;33:408-14.

5. Noyes FR, Mangine RE, Barber SD. The early treatment of motion complications after reconstruction of the anterior cruciate ligament. Clin Orthop Relat Res 1992;(277):217-28.

6. Shapiro MS, Freedman EL. Allograft reconstruction of the anterior and posterior cruciate ligaments after traumatic knee dislocation. Am J Sports Med 1995;23:580-7.

7. Lindenfeld TN, Wojtys EM, Husain A. Surgical treatment of arthrofibrosis of the knee. Instr Course Lect 2000;49:211-21.

8. Cosgarea AJ, DeHaven KE, Lovelock JE. The surgical treatment of arthrofibrosis of the knee. Am J Sports Med 1994;22:184-91.

9. Kukreja M, Kang J, Curry EJ, et al. Arthroscopic Lysis of Adhesions and Anterior Interval Release With Manipulation Under Anesthesia for Severe Post-traumatic Knee Stiffness: A Simple and Reproducible Step-by-Step Guide. Arthrosc Tech 2019;8:e429-35.

10. Carstensen SE, Feeley SM, Diduch DR. Manipulation Under Anesthesia With Lysis of Adhesions Is Effective in Arthrofibrosis After Sulcus-Deepening Trochleoplasty: A Prospective Study. Orthop J Sports Med 2019;7:2325967119864868.

11. Werner BC, Cancienne JM, Miller MD, et al. Incidence of manipulation under anesthesia or lysis of adhesions after arthroscopic knee surgery. Am J Sports Med 2015;43:1656-61.
12. Sprague NF, O'Connor RL, Fox JM. Arthroscopic treatment of postoperative knee fibroarthrosis. Clin Orthop Relat Res 1982;166:165-72.

13. Reider B, Belniak RM, Preiskorn D. Arthroscopic arthrolysis for flexion contracture following intraarticular reconstruction of the anterior cruciate ligament. Arthroscopy 1996;12:165-73.

14. Gu A, Michalak AJ, Cohen JS, Almeida ND, et al. Efficacy of Manipulation Under Anesthesia for Stiffness Following Total Knee Arthroplasty: A Systematic Review. J Arthroplasty 2018;33:1598-605.

15. Witvrouw E, Bellemans J, Victor J. Manipulation under anaesthesia versus low stretch device in poor range of motion after TKA. Knee Surg Sports Traumatol Arthrosc 2013;21:2751-8.

16. Dodds JA, Keene JS, Graf BK, et al. Results of knee manipulations after anterior cruciate ligament reconstructions. Am J Sports Med 1991;19:283-7.

17. Sassoon AA, Adigweme OO, Langford J, et al. Manipulation Under Anesthesia: A Safe and Effective Treatment for Posttraumatic Arthrofibrosis of the Knee. J Orthop Trauma 2015;29:e464-8.

18. Noyes FR, Berrios-Torres S, Barber-Westin SD, et al. Prevention of permanent arthrofibrosis after anterior cruciate ligament reconstruction alone or combined with associated procedures: a prospective study in 443 knees. Knee Surg Sports Traumatol Arthrosc 2000;8:196-206.

19. Fitzsimmons SE, Vazquez EA, Bronson MJ. How to treat the stiff total knee arthroplasty?: a systematic review. Clin Orthop Relat Res 2010;468:1096-106.

20. Mariani PP, Santori N, Rovere P, et al. Histological and structural study of the adhesive tissue in knee fibroarthrosis: a clinical-pathological correlation. Arthroscopy 1997;13:313-8.

21. Issa K, Banerjee S, Kester MA, et al. The effect of timing of manipulation under anesthesia to improve range of motion and functional outcomes following total knee arthroplasty. J Bone Joint Surg Am 2014;96:1349-57.

22. Thompson R, Novikov D, Cizmic Z, et al. Arthrofibrosis after total knee arthroplasty: pathophysiology, diagnosis, and management. Orthop Clin North Am 2019;50:269-79.

23. Ibrahim IO, Nazarian A, Rodriguez EK. Clinical Management of Arthrofibrosis: State of the Art and Therapeutic Outlook. JBJS Rev 2020;8:e1900223.

24. Brealey S, Northgraves M, Kottam L, et al. Surgical treatments compared with early structured physiotherapy in secondary care for adults with primary frozen shoulder: the UK FROST three-arm RCT. Health Technol Assess 
2020;24:1-162.

25. Hutton B, Wolfe D, Moher D, et al. Reporting guidance considerations from a statistical perspective: overview of tools to enhance the rigour of reporting of randomised trials and systematic reviews. Evid Based Ment Health 2017;20:46-52.

26. Altman DG, Moher D, Schulz KF. Improving the reporting of randomised trials: the CONSORT Statement and beyond. Stat Med 2012;31:2985-97.

27. Worsham J, Lowe WR, Copa D, et al. Subsequent Surgery for Loss of Motion After Anterior Cruciate Ligament Reconstruction Does Not Influence Function at 2 Years: A Matched Case-Control Analysis. Am J Sports Med 2019;47:2550-6.

28. Kalson NS, Borthwick LA, Mann DA, et al. International consensus on the definition and classification of fibrosis of the knee joint. Bone Joint J 2016;98-B:1479-88.

29. Riddle DL, Rothstein JM, Lamb RL. Goniometric reliability in a clinical setting. Shoulder measurements. Phys Ther 1987;67:668-73.

30. Irrgang JJ, Anderson AF, Boland AL, et al. Development and validation of the international knee documentation committee subjective knee form. Am J Sports Med 2001;29:600-13.

31. Anderson AF, Irrgang JJ, Kocher MS, et al. The International Knee Documentation Committee Subjective Knee Evaluation Form: normative data. Am J Sports Med 2006;34:128-35

32. Jia ZY, Zhang C, Zou Y, et al. Translation and validation of the simplified Chinese version of international knee documentation committee subjective knee form. Arch Orthop Trauma Surg 2018;138:1433-41.

33. Harris JD, Brand JC, Cote MP, et al. Research pearls: the significance of statistics and perils of pooling. part 1: clinical versus statistical significance. Arthroscopy

Cite this article as: Zhang L, Yan M, Chen S, Hua Y, Wang Z, $\mathrm{Xu}$ L, Fan S, Lin L, Cai B. Manipulation under anaesthesia versus lysis of adhesions for arthrofibrosis of the knee: a 6-month randomized, multicentre, non-inferiority comparative effectiveness protocol. Ann Palliat Med 2021;10(5):5908-5918. doi: 10.21037/apm-21-48
2017;33:1102-12.

34. Briggs KK, Lysholm J, Tegner Y, et al. The reliability, validity, and responsiveness of the Lysholm score and Tegner activity scale for anterior cruciate ligament injuries of the knee: 25 years later. Am J Sports Med 2009;37:890-7.

35. Huang H, Zhang D, Jiang Y, et al. Translation, Validation and Cross-Cultural Adaptation of a Simplified-Chinese Version of the Tegner Activity Score in Chinese Patients with Anterior Cruciate Ligament Injury. PLoS One 2016;11:e0155463.

36. Guo X, Wang K, Yu S, et al. Quality of life and its determinants in patients with treated acromegaly: a crosssectional nationwide study in china. J Clin Endocrinol Metab 2021;106:211-25.

37. Herdman M, Gudex C, Lloyd A, et al. Development and preliminary testing of the new five-level version of EQ-5D (EQ-5D-5L). Qual Life Res 2011;20:1727-36.

38. van de Graaf VA, Scholtes VA, Wolterbeek N, et al. Cost-effectiveness of Early Surgery versus Conservative Treatment with Optional Delayed Meniscectomy for Patients over 45 years with non-obstructive meniscal tears (ESCAPE study): protocol of a randomised controlled trial. BMJ Open 2016;6:e014381.

39. Graham JW, Olchowski AE, Gilreath TD. How many imputations are really needed? Some practical clarifications of multiple imputation theory. Prev Sci 2007;8:206-13.

40. Ipach I, Schäfer R, Lahrmann J, et al. Stiffness after knee arthrotomy: evaluation of prevalence and results after manipulation under anaesthesia. Orthop Traumatol Surg Res 2011;97:292-6.

41. Smith EL, Banerjee SB, Bono JV. Supracondylar femur fracture after knee manipulation: a report of 3 cases. Orthopedics 2009;32:18. 


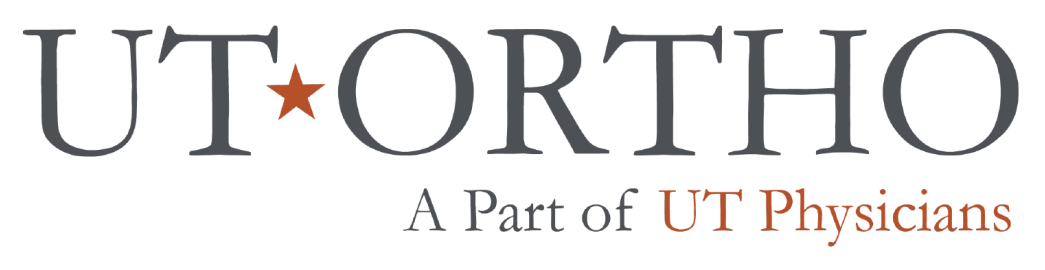

\section{ACL Reconstruction}

Post-Operative Rehabilitation Protocol

Ironman Sports Medicine Institute

1st Edition

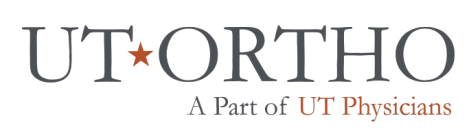




\section{ACL Reconstruction}

\section{PHASE 1 - ACUTE (0-6 Weeks)}

PHASE GOALS: PROTECT GRAFT, RESTORE AMBULATION \& ADL STATUS

RANGE OF MOTION *NO MENISCUS/CARTILAGE PROCEDURES

- 0-1 WEEK - FULL EXTENSION SLOWLY PROGRESS FLEXION TO 90 DEGREES

- 1+ WEEKS - MAINTAIN FULL EXTENSION \& GRADUALLY PROGRESS TO FULL FLEXION

WEIGHT BEARING

- 0-2 WEEKS - WEIGHT-BEARING AS TOLERATED

- 2+ WEEKS - FULL WEIGHT BEARING WITH SYMMETRICAL GAIT

BRACE \& CRUTCH USE

- 0-1 WEEK - BRACE LOCKED IN FULL EXTENSION

- 1-2 WEEKS - OPEN BRACE TO 60 DEG

- 2+ WEEKS - BRACE OPEN WITH GOOD QUAD CONTROL \& FULL EXTENSION; DC BRACE \& CRUTCHES WHEN GAIT IS NORMAL

\section{STRENGTHENING}

· QUAD SETS, STRAIGHT LEG RAISES, HIP ABDUCTION

- BALANCING, PRE-GAIT, CALF RAISES

\section{CRITERIA FOR FULL AMBULATION}

- > 0 DEG KNEE EXTENSION \& 90 DEG KNEE FLEXION

- $\quad 30$ STRAIGHT LEG RAISES WITHOUT LAG

- MINIMAL EFFUSION, PAIN, \& SYMMETRICAL GAIT WITHOUT A LIMP

- MD OR PT APPROVAL

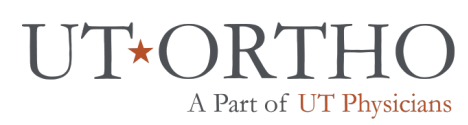




\section{ACL Reconstruction}

\section{PHASE 2 - STRENGTH \& JOGGING (6-12 Weeks)}

PHASE GOALS: IMPROVE STRENGTH \& INITIATE JOGGING PROGRAM RANGE OF MOTION

- 6+ WEEKS - SYMMETRICAL \& PAIN-FREE WITH OVERPRESSURE STRENGTHENING

· LEG PRESS, STEP-UPS, \& ROMANIAN DEADLIFTS (RDLS)

· SQUAT PROGRESSION (BODYWEIGHT SQUATS -> SINGLE LEG SQUATS)

- RESISTED HIP ABDUCTION LATERAL BAND WALKS

. CORE EXERCISES (PLANKS, V-UPS, SINGLE-LEG BRIDGING) CONDITIONING

· STATIONARY BIKING (MUST BE BEYOND 110 DEG KNEE FLEXION)

· ELLIPTICAL \& ROWING MACHINE

- SWIMMING (PROGRESS KICKING GRADUALLY \& PAIN-FREE)

\section{CRITERIA FOR JOGGING}

- PAIN LESS THAN 3 / 10 (WORST)

- WITHIN 2 DEG NORMAL KNEE EXTENSION \& 120 DEG KNEE FLEXION

- QUADRICEPS \& HAMSTRING STRENGTH $\geq 60 \%$ NORMAL

- LESS THAN 4cm DEFICIT ON SINGLE-LEg SQUAT (ANTERIOR REACH)

- AT LEAST 1 MINUTE OF SINGLE LEG SQUATS

- MD OR PT APPROVAL

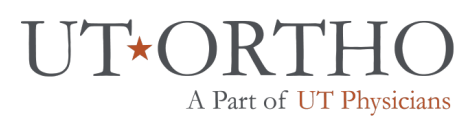




\section{ACL Reconstruction}

\section{PHASE 3 - AGILITY (12-20 Weeks)}

PHASE GOALS: INTRODUCE DYNAMIC \& POWER MOVEMENTS

RANGE OF MOTION

. 12+ WEEKS - MAINTAIN SYMMETRY \& PAIN-FREE OVERPRESSURE

\section{STRENGTHENING}

- GYM STRENGTHENING (SQUATS, DEADLIFTS, INITIATE OLYMPIC LIFTING)

- CORE EXERCISES (MOUNTAIN CLIMBERS, PLANKS, V-UPS)

- BIODEX FATIGUEING PROTCOLS

CONDITIONING

. BIKING, ELLIPTICAL, JOGGING, SWIMMING \& ROWING

\section{PLYOMETRICS \& LIGHT AGILITY}

· LADDER DRILLS, FOOTWORK AGILITIES

- BOX JUMPS (DOUBLE \& SINGLE LEG)

· HIGH INTENSITY PREDICTABLE AGILITY MOVEMENTS

\section{CRITERIA FOR HEAVY AGILITY}

- PAIN LESS THAN 2 / 10 (WORST)

- QUAD \& HAM STRENGTH $\geq 80 \%$ NORMAL; $\geq 50 \%$ H/Q RATIO FOR FEMALES

- AT LEAST 3 MINUTES OF SINGLE LEG SQUATS (RESISTED)

- $\leq 5$ ON LANDING ERROR SCORING SYSTEM (LESS)

- MD OR PT APPROVAL

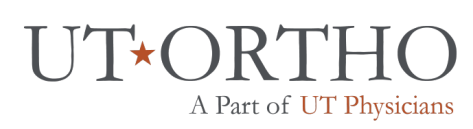




\section{ACL Reconstruction}

PHASE 4 - RETURN TO PLAY (24+ Weeks)

PHASE GOALS: INITIATE SPORTS SPECIFIC MOVEMENTS \& RETURN TO PLAY STRENGTHENING

- PROGRESS GYM STRENGTHENING (SQUATS, DEADLIFTS, \& OLYMPIC LIFTING)

- BIODEX FATIGUE PROTCOLS \& CORE EXERCISES

CONDITIONING

· JOGGING, BIKING, SWIMMING, \& INTERVAL SPRINT WORKOUTS

PLYOMETRICS \& AGILITY (2-3 DAYS/WEEK)

- MAX EFFORT BOX JUMPS (PROGRESS WITH ROTATION)

- LATERAL \& ROTATIONAL AGILITY

. UNPREDICTABLE CUTTING AGILITY \& CONTACT DRILLS

\section{CRITERIA FOR RETURN TO PLAY}

- PAIN LESS THAN 2 / 10 (WORST)

- >75/100 ON ACL-RSI SURVEY

- QUAD \& HAM STRENGTH $>90 \%$ NORMAL; $>60 \%$ H/Q RATIO FOR FEMALES

- $90 \%$ NORMAL ON SINGLE-LEG HOP TESTS

- 95\% NORMAL FIGURE OF 8, 5-10-5 PRO-AGILITY, \& S-L VERTICAL JUMP

- MD APPROVAL

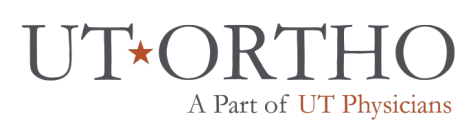

